\title{
A 160 Civarındaki Çift-Çift Çekirdeklerin Eşevreli Durum Yaklaşımı ile İncelenmesi
}

Geliş / Received: 29/01/2020

Melih Karataş ${ }^{1 *}$, İlyas İnci²

Revize / Revised: 05/03/2020

Kabul / Accepted: 06/03/2020

ÖZ

$\mathrm{Bu}$ çalışmada, kütle numarası 160 civarında bulunan çift-çift çekirdeklerin uyarılma enerji spektrumları incelenmiştir. U(5)-SU(3) bölgesi için Etkileşen Bozon Modeli Hamiltoniyeninin enerji özdeğerleri, düşük enerjili bantlar için Eşevreli Durumlar kullanılarak elde edilmiştir. Analitik olarak elde edilen enerji özdeğerleri, seçilen izotop zinciri için deneysel verilerle karşılaştırılmıştır. Deney ve teori arasında çok iyi bir uyum olduğu görülmüştür.

Anahtar Kelimeler- Çift-Çift Çekirdekler, Etkileşen Bozon Modeli, Eşevreli Durum Yaklaşımı, Ağır Çekirdekler

1*Sorumlu yazar iletişim: melihkaratasorg@gmail.com (https://orcid.org/0000-0001-7381-445X)

Fizik Bölümü, Çankırı Karatekin Üniversitesi, Uluyazı Kampüsü

2İletişim: inci.ilyas@gmail.com (https://orcid.org/0000-0002-2623-8186)

Fizik Bölümü, Çankırı Karatekin Üniversitesi, Uluyazı Kampüsü 


\title{
Investigation of Even-even Nuclei at around A 160 with the Coherent State Approximation
}

\begin{abstract}
In this study, excitation energy spectra of even-even nuclei having a mass number around 160 have been investigated. Energy eigenvalues of the Interacting Boson Model Hamiltonian for the U(5)-SU(3) region have been obtained for the low-lying energy bands by using Coherent States. Analytically obtained energy eigenvalues have been compared with the experimental data for some selected isotopic chain. It has been seen that there is a good agreement between theory and experiment.
\end{abstract}

Keywords- Even-even Nuclei, Interacting Boson Model, Coherent State Approximation, Heavy Nuclei 


\section{I.GİRIŞ}

Atom çekirdeklerinde, nükleon sayısının değişimine bağlı olarak gerçekleşen şekil faz geçişleri, nükleer yapı fiziğinin son yıllarda ilgilendiği en önemli konuların başında gelmektedir. Kütlesi az olan veya kapalı bir kabuğu olan çekirdekler, şekil olarak küresel simetrik bir yapıdadır. Proton ve nötron sayısının artışına bağlı olarak çekirdeğin şekli küresel yapıdan uzaklaşır. Bu durumun sonucu olarak da çekirdeğin elektrik kuadrupol momenti artar. Bu olgu, orta ve ağır çekirdekler için deneysel olarak da gösterilmiştir[1].

Bohr ve Mottelson'un oluşturduğu Kolektif Model küresel yapının dışında kalan nükleonların kolektif hareket ettiğini öngördüğü bir çalışmadır. Kolektif Model, orta ve ağır çekirdekler üzerinde oldukça başarılı bir modeldir[1]. Çekirdeklerde iki tip kolektif hareket durumu gözlenir. Bu hareketler titreşim ve dönmedir. Deformesi fazla olan çekirdeklerde simetri eksenine dik olan eksen etrafında bir dönme gözlenir. Eğer deforme durumu geçiciyse çekirdeğin herhangi bir andaki şekli eksensel asimetriktir ( $\gamma$-kararsız rotor)[2].

Atomik çekirdeklerin yapılarını açıklamada en başarılı ve Kolektif modele zıt olan modellerden biri Etkileşen Bozon Modeli (IBM)'dir. Bu modelin ilk versiyonunda, protonlar ve nötronlar arasında ayrım yapılmaz ve çiftlenen her nükleon bir bozon oluşturur ve en fazla 0 veya 2 açısal momentum taşıyabilir[3]. Son kapalı kabuğun dışında kalan nükleonlar proton veya nötron olduğu ayırt edilmeden, her iki nükleonun tek bir bozon oluşturacağı kabul edilerek işlem yapılır.

Elde edilen bozonlar sırasıyla s ve d bozonu olarak adlandırılır. s ve d bozonlarının manyetik izdüşümleri dikkate alındığında 6 farklı durumla karşılaşılır ve bu sistem, grup teori dilinde U(6) grubu ile ifade edilir. U(6)'nın alt grupları U(5), SU(3) ve $\mathrm{O}(6)$ olup sırasıyla küresel vibratör, deforme rotor ve gama kararsız rotor yapıları olarak adlandırılır[4].

Etkileşen Bozon Modeli zaman içinde farklı parametreler kullanılarak geliştirilmiştir[5]. Bu modelin ilk hali IBM-1olarak adlandırılmıştır. Orta-ağır ve ağır çift-çift çekirdeklerde oldukça başarlı olan IBM-1'de protonlar ve nötronlar simetrik kabul edilmiştir. Fakat düşük uyarılma $(<3 \mathrm{MeV})$ durumlarında uzak sonuçlar verir. Bu uyarılma durumlarının daha iyi elde edilebilmesi için protonlar ve nötronlar arasında ayrımın yapılması gerektiği görülmüştür ve geliştirilen model IBM-2 adını almıştır[6,7]. Her iki model de yalnızca çift sayıda proton ve nötron içeren çekirdekler için geçerlidir. Tek sayı nükleonlu çekirdeklerin yapısı ise, tek kalan fermiyonun çift sayıda nükleon içeren kor'a çiftlendiğinin kabul edildiği Etkileşen Bozon-Fermiyon Modeli (IBFM) ile açıklanır[3].

IBM hamiltoniyeni sadece limit durumlarda köşegenleştirilir, limitler arası geçiş bölgelerinde ise çözüm elde edebilmek için PHINT gibi paket programlar kullanmalıdır. Eşevreli Durum Yaklaşımı, geçiş bölgeleri arasında Hamiltoniyen hesaplanmasında yardımcı olur. Bu nedenle bu çalışmada SU(3) ile O(6) arasındaki geçiş bölgesinde yapı sergileyen A 160 civarındaki çekirdeklerin yapıları Eşevreli Durum Yaklaşımı (EDY) ile incelenmiştir. Bu kütle bölgesini EDY kullanarak inceleyen çalışma literatürde olmamasına karşın Değişken Eylemsizlik Momenti (VMI) kullanılarak bu kütle bölgesi incelenmiştir [8]. İncelen izotopların uyarılma enerji spektrumu ve durumlar arası elektrik kuadrupol geçiş oranları elde edilmiştir. Teorik sonuçların, deneysel verilerle oldukça uyumlu olduğu görülmüştür.

\section{II.MATERYAL VE METOD}

Eşevreli Durum Yaklaşımında incelenen çekirdeklerin enerji özdeğerleri, Etkileşen Bozon Modeli Hamiltoniyeni ile Kolektif Model parametreleri $(\beta, \gamma)$ kullanılarak yazılan özfonksiyonlar sayesinde hesaplanır. $\mathrm{Bu}$ nedenle bu yaklaşım, IBM ile Kolektif model arasında köprü görevi görür. İncelenen U(5) - SU(3) bölgesi için multipol momentler cinsinden IBM hamiltoniyeni aşağıdaki gibi verilir [9],

$$
\widehat{H}=\kappa_{3} \hat{Q} \cdot \hat{Q}-\kappa^{\prime} \hat{L} \cdot \hat{L}+\kappa^{\prime \prime} P^{\dagger} \cdot \tilde{P}
$$


Burada Q, L ve P sırasıyla kuadupol moment, açısal momentum ve çiftlenim operatörleri olup s ve d bozon operatörleri cinsinden şöyle tanımlanır [9],

$$
\begin{aligned}
& \hat{Q}=\left(d^{+} s+s^{+} d\right)+\chi \widehat{T}_{2} \\
& \hat{L}=\sqrt{10} \widehat{T}_{1} \\
& \tilde{P}=\frac{1}{2}(\tilde{d} \cdot \tilde{d}-s \cdot s) \\
& \widehat{T}_{\ell=}\left(d^{\dagger} \tilde{d}\right)^{(\ell)} ; \ell=0,1,2,3,4
\end{aligned}
$$

$\kappa_{3}$ ve $\kappa^{\prime}$, taban durum bandı ile gamma band $2^{+}$enerjilerine bağlı olup

$$
\begin{aligned}
& \kappa_{3}=\frac{E_{\gamma}\left(2^{+}\right)-E_{g}\left(2^{+}\right)}{3(1-2 N)} \\
& \kappa^{\prime}=-\frac{3}{8} \kappa_{3}-\frac{1}{6} E_{\gamma}\left(2^{+}\right)
\end{aligned}
$$

ifadesiyle belirlenir. $\kappa$ "ise, tamamıla serbest parametredir. Kuadrupol operatöründe bulunan düzen parametresi $\chi$ ise $\mathrm{U}(5)$ limitinde 0 ve $\mathrm{SU}(3)$ limitinde $-\sqrt{7 / 4}$ değerini alır[10,11].

Kapalı kabuk dışında 2N tane nükleondan yani $\mathrm{N}$ tane bozondan oluşan bir çift-çift çekirdek sisteminin taban durumu, bozon yoğunlaşması olarak adlandırılan $\mid 0>$ durumu üzerine $\mathrm{N}$ defa taban durum için bozon yaratma operatörü $b_{g}^{\dagger}$ uygulanarak elde edilir,

$$
b_{g}^{\dagger}\left(a_{\mu}\right)=s^{\dagger}+\sum_{\mu} a_{\mu} d_{\mu}^{\dagger}
$$

$\mathrm{N}$ tane değerlik bozon içeren sistemin taban durumu bozon yoğunlaşması adıyla tanımlanır. IBM'e göre sadece s ve d bozonları sistemde olduğu için bozon üretme operatörleri, bu bozonlara karşılık gelen operatörlerin ortogonal kombinasyonları ile verilir. Taban durumu üreten bozon operatörü aşağıdaki denklemde verilmiştir.

$$
b_{g}^{\dagger}(\beta, \gamma)=\frac{1}{\sqrt{1+\beta^{2}}}\left[s^{\dagger} \beta \cos \gamma d_{0}^{\dagger}+\frac{\beta}{\sqrt{2}} \sin \gamma\left(d_{2}^{\dagger}+d_{-2}^{\dagger}\right)\right]
$$

Burada $\beta$ ve $\gamma$, Bohr Hamiltoniyenindeki iç kolektif değişken anlamındadır. $\mathrm{N}$ tane bozondan oluşan sistemin taban durumu yukarıda tanımlanan bozon operatörünün $\mathrm{N}$ defa $|0\rangle$ ile gösterilen vakum durumuna uygulanmasiyla elde edilir.

$$
|\beta, \gamma\rangle^{g}=\frac{1}{\sqrt{N !}}\left(b_{g}^{\dagger}(\beta, \gamma)\right)^{N}|0\rangle^{g}
$$

İç uyarılmalar taban durumundaki, bozon sayısının değişmesine neden olacaktır. Böylece durum fonksiyonundaki, taban durum operatörlerinden bir tanesi uyarılmayı sağlayan başka bir operatörlere yer değiştirecektir[10]. İlk uyarılmayı sağlayan operatör $\beta$ titreşimini üretir. Aşağıdaki operatör ilk uyarılmayı sağlayan operatördür.

$$
b_{\beta v}^{\dagger}(\beta, \gamma)=\frac{1}{\sqrt{1+\beta^{2}}}\left[-\beta s^{\dagger} \cos \gamma d_{0}^{\dagger}+\frac{1}{\sqrt{2}} \sin \gamma\left(d_{2}^{\dagger}+d_{-2}^{\dagger}\right)\right]
$$

$\beta$ titreşim bandı durumları ise (12) ve (13) numaralı denklemlerle elde edilir.

$$
|\beta, \gamma\rangle^{g}==\frac{1}{\sqrt{N !}} b_{\beta v}^{\dagger}(\beta, \gamma) b_{g . s .}^{\dagger}(\beta, \gamma) I_{g . s .}(\beta, \gamma)
$$




$$
|\beta, \gamma\rangle^{g}==\frac{1}{\sqrt{(N-1) !}} b_{\beta v}^{\dagger}(\beta, \gamma)\left(b_{g}^{\dagger}(\beta, \gamma)\right)^{N-1}|0\rangle
$$

Bir sonraki uyarılma durumu ise $\gamma$ titreşim bandı olup buna karşıllk gelen üretici operatör ve sistemin durumu(14) ve (15) ile verilir[9].

$$
\begin{aligned}
& b_{\gamma v}^{\dagger}(\beta, \gamma)=\left[-\sin \gamma d_{0}^{\dagger}+\frac{1}{\sqrt{2}} \cos \gamma\left(d_{2}^{\dagger}+d_{-2}^{\dagger}\right)\right] \\
& |\beta, \gamma\rangle^{\gamma}==\frac{1}{\sqrt{N !}} b_{\gamma v}^{\dagger}(\beta, \gamma) b_{g . s .}^{\dagger}(\beta, \gamma) I_{g}(\beta, \gamma) \\
& |\beta, \gamma\rangle^{\gamma}=\frac{1}{\sqrt{(N-1) !}} b_{\beta v}^{\dagger}(\beta, \gamma)\left(b_{g}^{\dagger}(\beta, \gamma)\right)^{N-1}|0\rangle \\
& \left|N ; a_{\mu}\right\rangle^{g}=N_{N}\left(b_{g}^{\dagger}\left(a_{\mu}\right)\right)^{N}|0\rangle
\end{aligned}
$$

Burada $N_{N}$ normalizasyon sabiti, $a_{\mu}(\mu=-1,-2,0,1,2)$ beş reel değişkeni olup Bohr şekil parametreleriyle orantılıdır[12,13]. Taban durum enerjisi ise Hamiltoniyenin beklenen değerinin hesaplanması ile elde edilir.

$$
E_{g}\left(N, a_{\mu}\right)={ }^{g}\left\langle N ; a_{\mu}|\widehat{H}| N ; a_{\mu}\right\rangle^{g}
$$

Taban durum bandından daha yüksek enerjili bandları elde edebilmek için taban durumdaki bir bozon yaratma operatörü, bu operatör ile ortagonal olan başka bir operatör ile yer değiştirmelidir. $\beta$ ve $\gamma$ titreşimlerini verecek bozon yaratma operatörleri sırasıyla,

$$
E_{\gamma}\left(N, a_{\mu}\right)={ }^{\gamma}\left\langle N ; a_{\mu}|\widehat{H}| N ; a_{\mu}\right\rangle^{\gamma}
$$

Beta bandının enerji ifadesi de bu șekilde tanımlanır.

$$
\begin{aligned}
& E_{\beta}\left(N, a_{\mu}\right)={ }^{\beta}\left\langle N ; a_{\mu}|\widehat{H}| N ; a_{\mu}\right\rangle^{\beta} \\
& \mathrm{I}=\frac{\left(\mathrm{dL}_{\mathrm{x}} / \mathrm{da}_{1}\right)^{2}}{\mathrm{~d}^{2} E_{g} / \mathrm{da}_{1}^{2}}
\end{aligned}
$$

Burada I, çekirdeğin eylemsizlik momentidir. Aynı enerji bandında farklı açısal momentumlu seviyelerin enerjileri ise sırasıyla taban durumu, $\beta$ ve $\gamma$ bantları için şu şekildedir.[14,15]

$$
\begin{aligned}
& E_{g}^{e x c}(N, L)=\frac{L(L+1)}{2 \mathrm{I}} \\
& E_{\beta}^{e x c}(N, L)=\frac{L(L+1)}{2 \mathrm{I}}+\left(E_{\beta}-E_{g}\right) \\
& E_{\gamma}^{e x c}(N, L)=\frac{L(L+1)}{2 \mathrm{I}}+\left(E_{\gamma}-E_{\beta}\right) \\
& I_{g}=\frac{-42 \mathrm{Nn} \beta^{2}\left(1+\beta^{2}\right)}{A \cdot \kappa^{\prime}+B \kappa^{\prime \prime}+C \cdot k_{3}} \\
& A=42\left(1+\beta^{2}\right)\left(1+2(-1+\mathrm{Nn}) \beta^{2}\right) \\
& B=7(-1+\mathrm{Nn}) \beta^{2}\left(-1+\beta^{2}\right) \\
& C=\left[28+2 \sqrt{14}(-1+\mathrm{Nn}) \beta \chi-6 \sqrt{14}(-1+\mathrm{Nn}) \beta^{3} \chi-7 \chi^{2}+2(-1+\mathrm{Nn}) \beta^{4} \chi^{2}-\beta^{2}\left(28+5 \chi^{2}+\right.\right. \\
& \left.\left.2 \mathrm{Nn}\left(-28+\chi^{2}\right)\right)\right]
\end{aligned}
$$


e-ISSN: 2458-7575 (http://dergipark.gov.tr/bseufbd)

\section{SONUÇLAR VE TARTIŞMA}

Elde edilen sonuçları kullanarak, seçilen bir çekirdeğin uyarılma spektrumunu elde etmek için Denk.(1)'de bulunan serbest parametrelerin belirlenmesi gerekir. $\kappa_{3}$ ve $\kappa^{\prime}$ parametreleri, tamamen serbest olmayıp Denk.(6,7) ile verildiği gibi deneysel verilerden elde edilir. $\kappa^{\prime \prime}$ ise tamamen serbest olup, deneysel verilerle en iyi uyumu elde etmek için ayarlanır. Bunun için ilk önce seçilen bir izotopun deneysel olarak belirlenmiş uyarılma enerjiler alınır. Daha sonra bu enerjileri en iyi tahmin eden parametre seti, deney ve teori arasındaki fark minimum olacak şekilde belirlenir.

Diğer bir nicelik ise her çekirdek için farklı olan bozon sayısı (N)'dir. Toplam bozon sayısı, son kapalı kabuğa göre işlem yapılarak proton ve nötron sayıları toplanıp ikiye bölünerek bulunur. Her bir çekirdek için bulunan bozon sayısı Tablo.1 de N sütununda gösterilmiştir. Proton ve nötron sayılarının oluşturacağı bozon sayısı hesabı, sihirli sayılara $(2,8,20,28,50,82$ ve 126) yakınlık kuralına göre yapılmaktadır. Örneğin; ${ }_{64}^{152} G d$ 'un 64 protonu ve 88 nötronu bulunmaktadır. Başta proton ve nötron sayısının hangi sihirli sayıya yakın olduğu hesaplanarak işlem yapılmaktadır. 88 nötronun en yakın olduğu sayı 82, 64 protonun ise 50 sihirli sayısıdır. Sihirli sayılar kapalı kabukta bulunabilecek maksimum nükleon sayılarını göstermektedir. Bozon sayısının hesabı ise şu şekildedir. Nötronun çekirdeğe bozon katkısı $\frac{(88-82)}{2}=3$, protonun çekirdeğe bozon katkısı ise $\frac{(64-50)}{2}=7$ 'dir. Buradan ${ }_{64}^{152} G d$ için toplam bozon sayısı $(\mathrm{N}), 7+3=10$ olarak bulunur.

Tablo 1. İzotopların deneysel uyarılma enerjilerini veren Hamiltoniyen parametreleri.

\begin{tabular}{|c|c|c|c|c|c|}
\hline İzotop & $\mathbf{N}$ & $\beta$ & $\kappa_{3}$ & $\boldsymbol{\kappa}^{\prime}$ & $\boldsymbol{\kappa}^{\prime \prime}$ \\
\hline${ }_{60}^{150} \mathrm{Nd}$ & 9 & 1.2733 & -18.45 & -13.8 & -15.2 \\
\hline${ }_{64}^{152} G d$ & 10 & 0.8216 & -13.42 & -52.3 & 3.9 \\
\hline${ }_{62}^{152} \mathrm{Sm}$ & 10 & 1.3188 & -16.91 & -13.82 & -23.9 \\
\hline${ }_{66}^{154} D y$ & 11 & 0.8024 & -11 & -51.54 & 7.7 \\
\hline${ }_{64}^{54} G d$ & 11 & 1.2566 & -13.86 & -15.3 & -12 \\
\hline${ }_{62}^{154} \mathrm{Sm}$ & 11 & 1.3936 & -21.56 & -5.58 & -19.7 \\
\hline${ }_{66}^{156} \mathrm{Dy}$ & 12 & 1.1561 & -10.91 & -18.87 & 0.9 \\
\hline${ }_{68}^{156} \mathrm{Er}$ & 10 & 0.8235 & -10.26 & -53.65 & 51 \\
\hline${ }_{64}^{156} \mathrm{Gd}$ & 12 & 1.2742 & -15.43 & -9.03 & 5.8 \\
\hline${ }_{66}^{158} \mathrm{Dy}$ & 13 & 1.1859 & -11.29 & -12.24 & 19.4 \\
\hline${ }_{64}^{158} \mathrm{Gd}$ & 13 & 1.2629 & -14.76 & -7.71 & 13.9 \\
\hline${ }_{68}^{160} \mathrm{Er}$ & 12 & 1.1292 & -10.55 & -17 & 23.7 \\
\hline${ }_{64}^{160} G d$ & 12 & 1.1669 & -11.27 & -8.31 & 43.8 \\
\hline${ }_{66}^{162} D y$ & 15 & 1.1342 & -9.28 & -9.96 & 47.6 \\
\hline${ }_{68}^{162} \mathrm{Er}$ & 13 & 1.1484 & 10.64 & -13.01 & 30.08 \\
\hline${ }_{66}^{164} D y$ & 16 & 1.1830 & -7.38 & -9.56 & 16.2 \\
\hline${ }_{68}^{164} \mathrm{Er}$ & 14 & 1.1297 & -9.48 & -11.67 & 42.5 \\
\hline${ }_{70}^{166} \mathrm{Yb}$ & 13 & 1.1644 & -11.06 & -12.91 & 25.6 \\
\hline${ }_{72}^{168} \mathrm{Hf}$ & 12 & 1.1239 & -10.88 & -16.59 & 25.9 \\
\hline${ }_{70}^{168} Y b$ & 14 & 1.1857 & -11.05 & -10.52 & 26.7 \\
\hline
\end{tabular}

Tablo 1'de verilen parametre setleri kullanılarak elde edilen uyarılma enerji spektrumları, elde edilen uyumun daha net görülebilmesi için Şekil 1-6’da en düşük enerjili üç band için verilmiştir. Bu şekillerde tüm 
e-ISSN: 2458-7575 (http://dergipark.gov.tr/bseufbd)

enerjiler keV cinsinden olup siyah renkle gösterilen düzeyler teorik sonuçları, mavi ile gösterilen düzeyler ise deneysel verileri belirtmektedir. Her durumun spin-paritesi, teorik düzeylerin üstünde yer almaktadır ve aynı spin-pariteli deneysel düzeyler, noktalı çigilerle teorik olana bağlanmıştır. Bu şekiller incelendiğinde ${ }_{60}^{150} N d$ hariç

taban durum (GS-band) bandında $8^{+}$açısal momentumlu duruma kadar teori ile deney çok uyumludur. Durumların açısal momentum değeri arttıkça teorik sonuçların daha hızlı arttı̆̆ı görülmektedir. Bununla birlikte tüm izotoplar için $\gamma$-bandında tahminler oldukça iyi, taban durum bandında gözlenen hızlı artış bu bantta görülmemektedir. $\beta$ bandında ise deneysel verilerin kısmen az olması, karşılaştırma yapmayı güçleştirmektedir. $\mathrm{Bu}$ şekiller dışında, diğer tüm izotoplar için hesaplanan enerji değerlerinin deneysel verilerle karşılaştırılması, her bir band için Tablo 2-4'te verilmiştir.

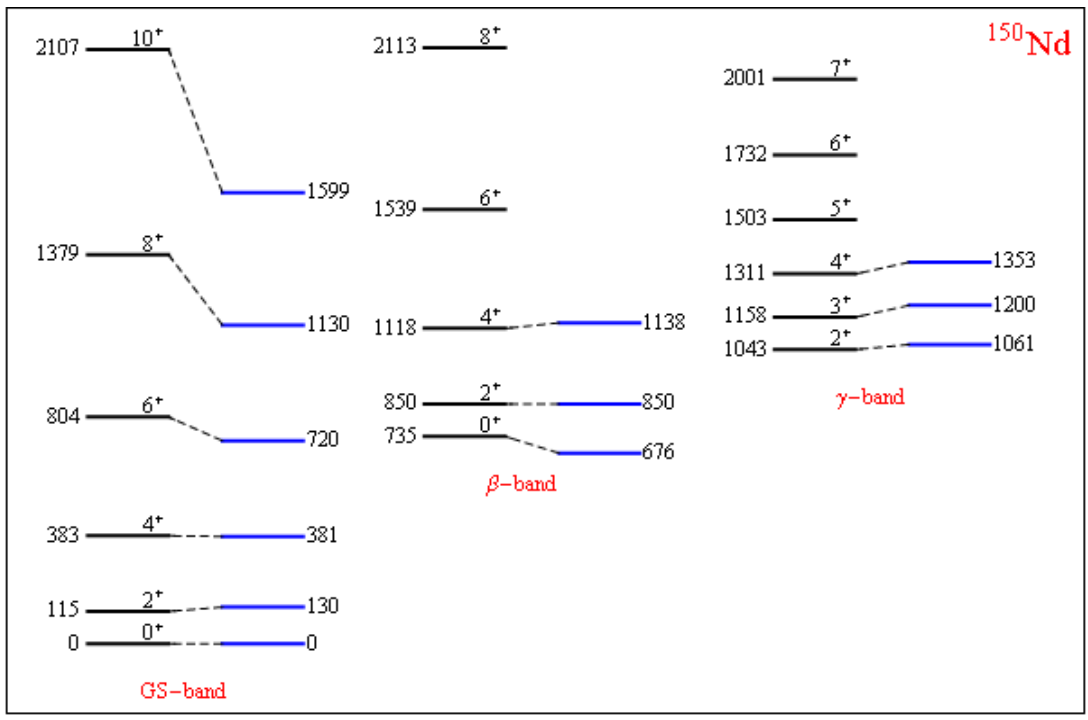

Şekil 1. ${ }_{60}^{150} \mathrm{Nd}$ için teorik (siyah) sonuçların deneysel (mavi) verilerle [16] karşılaştırılması. Enerjiler keV cinsindendir.

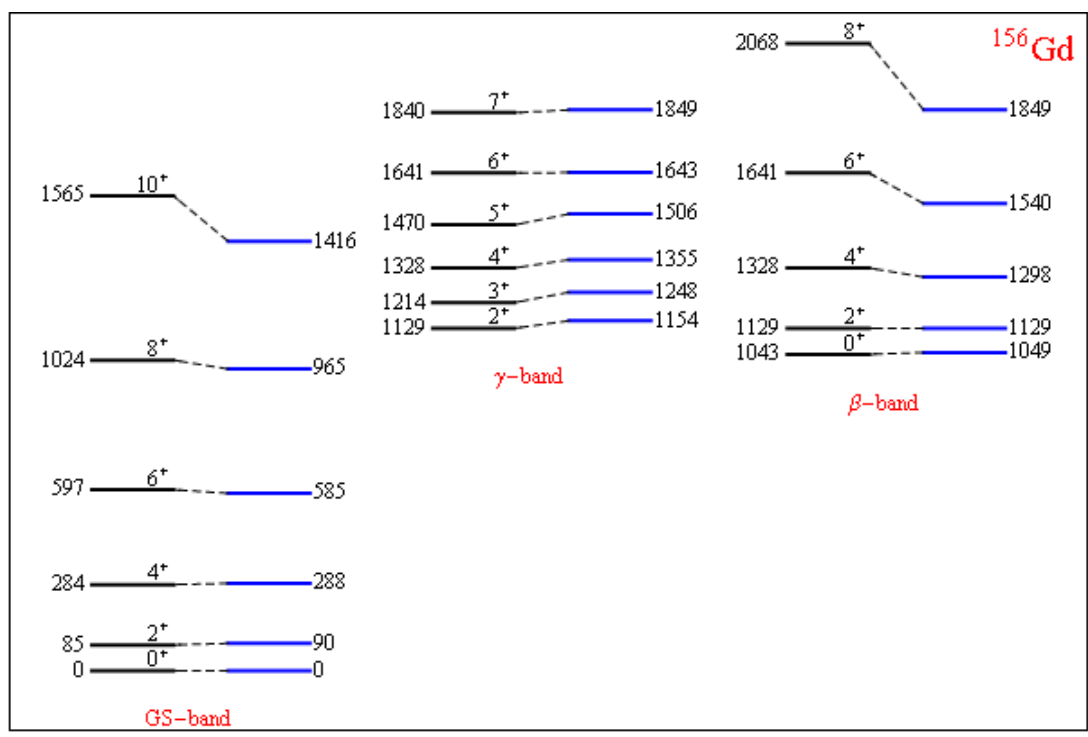


Şekil 2. ${ }_{64}^{156} G d$ için teorik (siyah) sonuçların deneysel (mavi) verilerle [17] karşılaştırılması. Enerjiler keV cinsindendir.

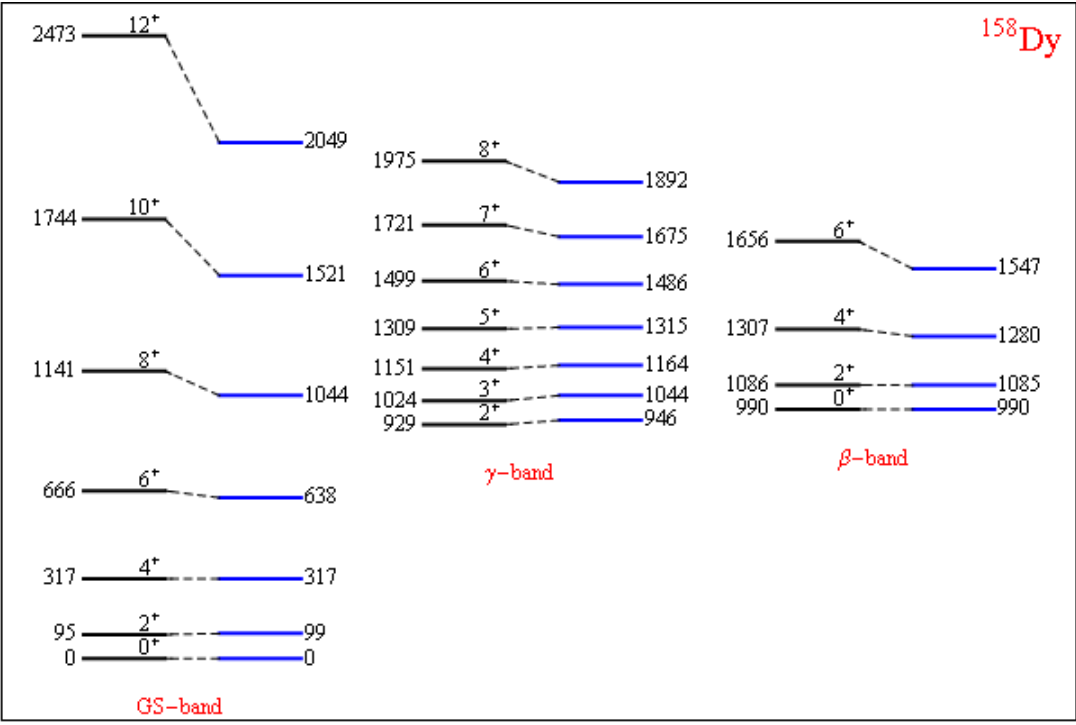

Şekil 3. ${ }_{66}^{158} D y$ için teorik (siyah) sonuçların deneysel (mavi) verilerle [18] karşılaştırılması. Enerjiler keV cinsindendir.

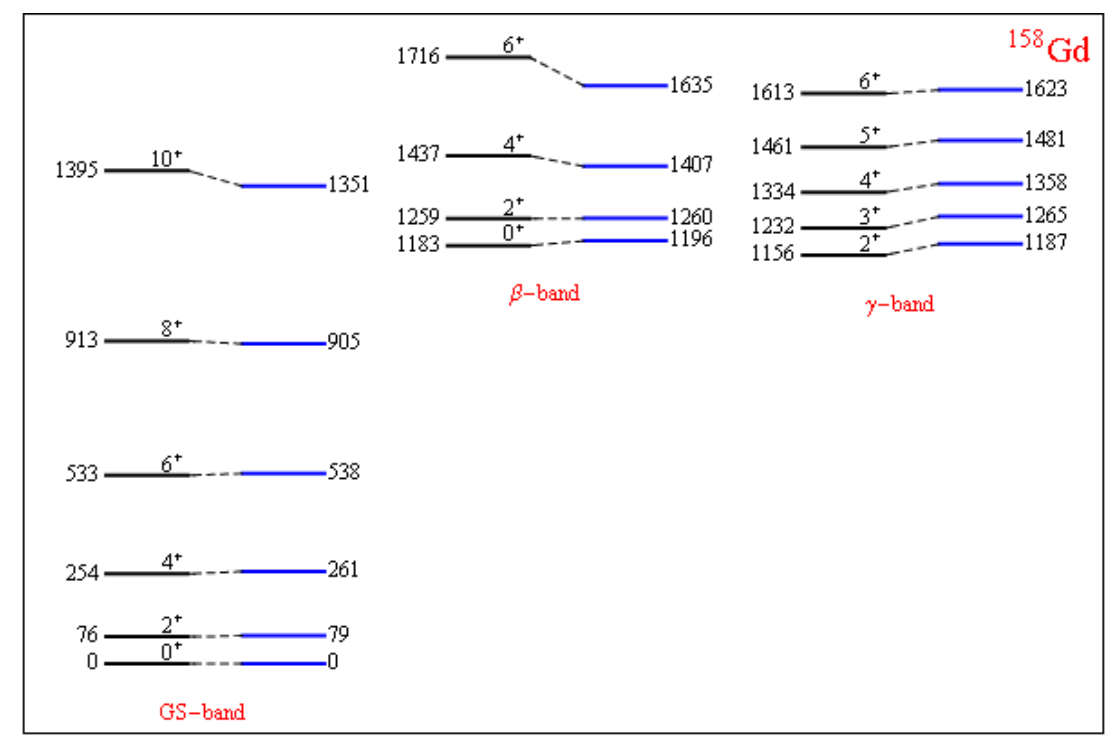

Şekil 4. ${ }_{64}^{158} G d$ için teorik (siyah) sonuçların deneysel (mavi) verilerle [18] karşılaştırılması. Enerjiler keV cinsindendir. 


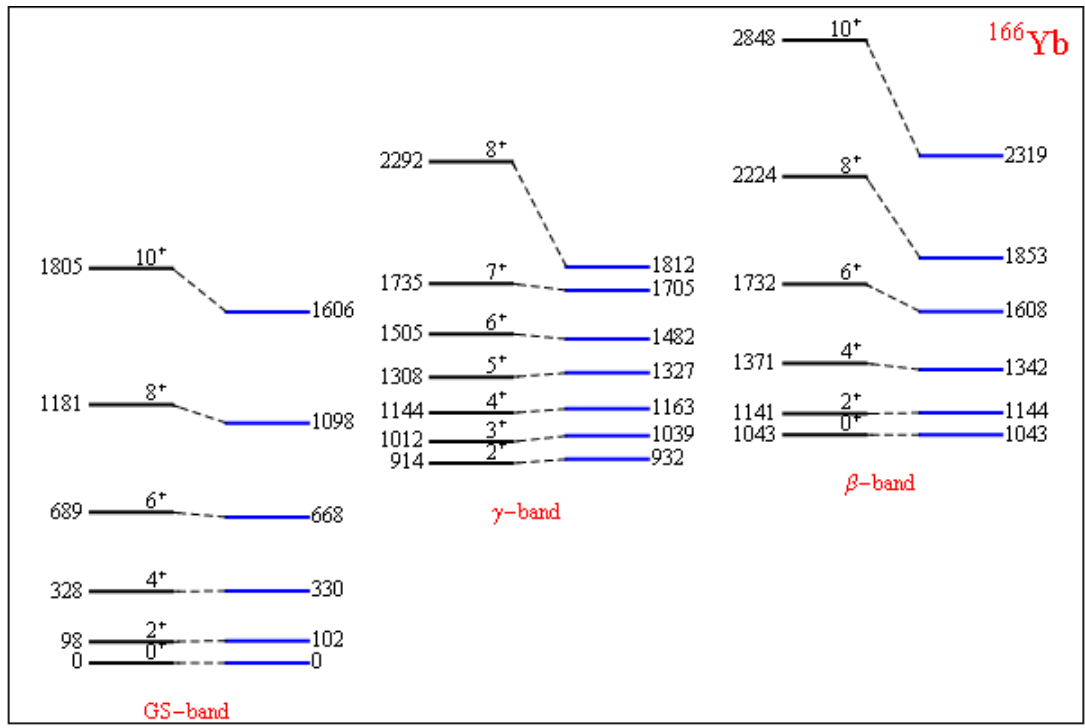

Şekil 5. ${ }_{70}^{166} \mathrm{Yb}$ için teorik (siyah) sonuçların deneysel (mavi) verilerle [19] karşılaştırılması. Enerjiler keV cinsindendir.

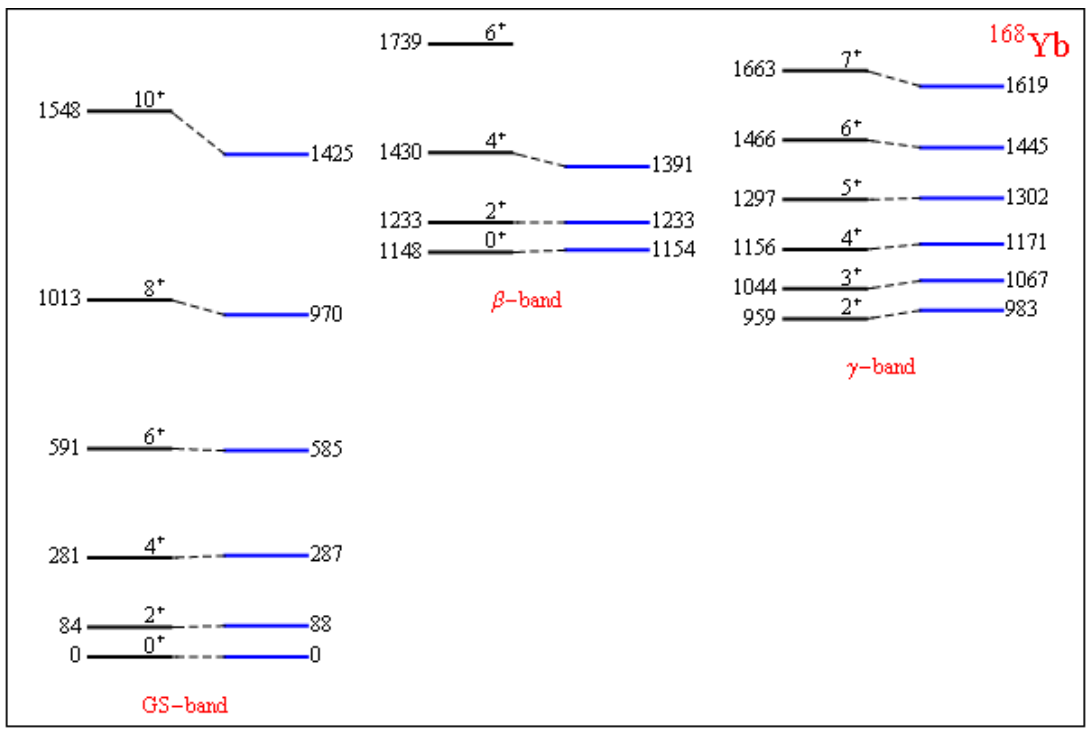

Şekil 6. ${ }_{70}^{168} \mathrm{Yb}$ için teorik (siyah) sonuçların deneysel (mavi) verilerle [20] karşılaştırılması. Enerjiler keV cinsindendir. 
e-ISSN: 2458-7575 (http://dergipark.gov.tr/bseufbd)

Tablo 2. Taban durumu için hesaplanan teorik sonuçlar ve deneysel veriler[16-26] (keV cinsinden)

\begin{tabular}{|c|c|c|c|c|c|c|c|c|c|c|}
\hline \multirow{2}{*}{ İzotop } & \multicolumn{2}{|c|}{$E\left(2_{1}^{+}\right)$} & \multicolumn{2}{|c|}{$E\left(4_{1}^{+}\right)$} & \multicolumn{2}{|c|}{$E\left(6_{1}^{+}\right)$} & \multicolumn{2}{|c|}{$E\left(8_{1}^{+}\right)$} & \multicolumn{2}{|c|}{$E\left(10_{1}^{+}\right)$} \\
\hline & Teori & Deney & Teori & Deney & Teori & Deney & Teori & Deney & Teori & Deney \\
\hline${ }_{60}^{150} \mathrm{Nd}$ & 115 & 130 & 383 & 381 & 804 & 720 & 1379 & 1130 & 2107 & 1599 \\
\hline${ }_{64}^{152} G d$ & 343 & 344 & 1143 & 755 & 2400 & 1227 & 4114 & 1746 & 6286 & 2300 \\
\hline${ }_{62}^{152} \mathrm{Sm}$ & 117 & 122 & 389 & 366 & 818 & 707 & 1402 & 1125 & 2142 & 1609 \\
\hline${ }_{66}^{154} D y$ & 334 & 334 & 1114 & 747 & 2340 & 1224 & 4011 & 1748 & 6128 & 2304 \\
\hline${ }_{64}^{154} G d$ & 119 & 123 & 396 & 371 & 831 & 718 & 1425 & 1144 & 2177 & 1637 \\
\hline${ }_{62}^{154} \mathrm{Sm}$ & 80 & 82 & 266 & 267 & 558 & 543 & 957 & 903 & 1462 & 1333 \\
\hline${ }_{66}^{156} \mathrm{Dy}$ & 133 & 138 & 445 & 404 & 934 & 771 & 1601 & 1216 & 2446 & 1725 \\
\hline${ }_{68}^{156} \mathrm{Er}$ & 346 & 344 & 1153 & 797 & 2422 & 1340 & 4152 & 1959 & 6344 & 2635 \\
\hline${ }_{64}^{156} G d$ & 85 & 90 & 284 & 288 & 597 & 585 & 1024 & 965 & 1565 & 1416 \\
\hline${ }_{66}^{158} \mathrm{Dy}$ & 95 & 99 & 317 & 317 & 666 & 638 & 1141 & 1044 & 1744 & 1521 \\
\hline${ }_{64}^{158} G d$ & 76 & 79 & 254 & 261 & 533 & 538 & 913 & 905 & 1395 & 1351 \\
\hline${ }_{68}^{160} \mathrm{Er}$ & 121 & 126 & 405 & 390 & 850 & 766 & 1457 & 1229 & 2226 & 1761 \\
\hline${ }_{64}^{160} G d$ & 72 & 75 & 239 & 248 & 501 & 514 & 859 & 871 & 1313 & 1308 \\
\hline${ }_{66}^{162} D y$ & 77 & 81 & 258 & 266 & 541 & 548 & 927 & 921 & 1417 & 1374 \\
\hline${ }_{68}^{162} \mathrm{Er}$ & 98 & 102 & 327 & 329 & 687 & 667 & 1177 & 1097 & 1799 & 1602 \\
\hline${ }_{66}^{164} D y$ & 71 & 73 & 238 & 242 & 501 & 501 & 858 & 844 & 1311 & 1261 \\
\hline${ }_{68}^{164} \mathrm{Er}$ & 88 & 91 & 293 & 299 & 615 & 614 & 1054 & 1025 & 1610 & 1518 \\
\hline${ }_{70}^{166} \mathrm{Yb}$ & 98 & 102 & 328 & 330 & 689 & 668 & 1181 & 1098 & 1805 & 1606 \\
\hline${ }_{72}^{168} \mathrm{Hf}$ & 120 & 124 & 399 & 386 & 837 & 757 & 1435 & 1213 & 2192 & 1736 \\
\hline${ }_{70}^{168} \mathrm{Yb}$ & 84 & 88 & 281 & 287 & 591 & 585 & 1013 & 970 & 1548 & 1425 \\
\hline
\end{tabular}


e-ISSN: 2458-7575 (http://dergipark.gov.tr/bseufbd)

Tablo 3. Beta için hesaplanan teorik sonuçlar ve deneysel veriler[16-26] (keV cinsinden)

\begin{tabular}{|c|c|c|c|c|c|c|c|c|c|c|}
\hline \multirow{2}{*}{ İzotop } & \multicolumn{2}{|c|}{$E\left(0_{2}^{+}\right)$} & \multicolumn{2}{|c|}{$E\left(2_{2}^{+}\right)$} & \multicolumn{2}{|c|}{$E\left(4_{2}^{+}\right)$} & \multicolumn{2}{|c|}{$E\left(6_{2}^{+}\right)$} & \multicolumn{2}{|c|}{$E\left(8_{2}^{+}\right)$} \\
\hline & Teori & Deney & Teori & Deney & Teori & Deney & Teori & Deney & Teori & Deney \\
\hline${ }_{60}^{150} \mathrm{Nd}$ & 735 & 676 & 850 & 850 & 1118 & 1138 & 1539 & - & 2113 & - \\
\hline${ }_{64}^{152} G d$ & 587 & 615 & 930 & 931 & 1730 & 1282 & 2987 & 1668 & 4702 & 2139 \\
\hline${ }_{62}^{152} \mathrm{Sm}$ & 684 & 685 & 801 & 810 & 1074 & 1023 & 1502 & 1310 & 2086 & 1667 \\
\hline${ }_{66}^{154} D y$ & 571 & 660 & 905 & 905 & 1685 & 152 & 2910 & 1658 & 4582 & 2163 \\
\hline${ }_{64}^{154} G d$ & 680 & 681 & 798 & 815 & 1075 & 1048 & 1511 & 1366 & 2105 & 1757 \\
\hline${ }_{62}^{154} \mathrm{Sm}$ & 1100 & 1100 & 1180 & 1178 & 1365 & 1371 & 1658 & 1577 & 2057 & - \\
\hline${ }_{66}^{156} \mathrm{Dy}$ & 676 & 675 & 809 & 829 & 1121 & 1089 & 1610 & 1438 & 2277 & 1859 \\
\hline${ }_{68}^{156} \mathrm{Er}$ & 874 & 930 & 1220 & 1220 & 2027 & 1546 & 3296 & - & 5026 & - \\
\hline${ }_{64}^{156} G d$ & 1043 & 1049 & 1129 & 1129 & 1328 & 1298 & 1641 & 1540 & 2068 & 1849 \\
\hline${ }_{66}^{158} D y$ & 990 & 990 & 1086 & 1085 & 1307 & 1280 & 1656 & 1547 & 2132 & - \\
\hline${ }_{64}^{158} G d$ & 1183 & 1196 & 1259 & 1260 & 1437 & 1407 & 1716 & 1635 & 2096 & - \\
\hline${ }_{68}^{160} \mathrm{Er}$ & 894 & 894 & 1016 & 1007 & 1299 & - & 1744 & - & 2351 & - \\
\hline${ }_{64}^{160} G d$ & 1380 & 1379 & 1451 & 1436 & 1618 & - & 1881 & - & 2239 & - \\
\hline${ }_{66}^{162} D y$ & 1376 & 1400 & 1453 & 1543 & 1634 & 1574 & 1917 & 1767 & 2303 & 1985 \\
\hline${ }_{68}^{162} \mathrm{Er}$ & 1073 & 1087 & 1171 & 1171 & 1400 & 1369 & 1760 & - & 2250 & - \\
\hline${ }_{66}^{164} D y$ & 861 & - & 932 & - & 1099 & - & 1362 & - & 1719 & - \\
\hline${ }_{68}^{164} \mathrm{Er}$ & 1226 & 1246 & 1314 & 1315 & 1519 & 1469 & 1841 & 1707 & 2280 & 2069 \\
\hline${ }_{70}^{166} \mathrm{Yb}$ & 1043 & 1043 & 1141 & 1144 & 1371 & 1342 & 1732 & 1608 & 2224 & 1853 \\
\hline${ }_{72}^{168} \mathrm{Hf}$ & 939 & 942 & 1059 & 1058 & 1338 & 1284 & 1776 & - & 2374 & - \\
\hline${ }_{70}^{168} Y b$ & 1148 & 1154 & 1233 & 1233 & 1430 & 1391 & 1739 & - & 2162 & - \\
\hline
\end{tabular}


e-ISSN: 2458-7575 (http://dergipark.gov.tr/bseufbd)

Tablo 4. Gama için hesaplanan teorik sonuçlar ve deneysel veriler[16-26] (keV cinsinden)

\begin{tabular}{|c|c|c|c|c|c|c|c|c|c|c|}
\hline \multirow{2}{*}{ İzotop } & \multicolumn{2}{|c|}{$E\left(2_{1}^{+}\right)$} & \multicolumn{2}{|c|}{$E\left(3_{1}^{+}\right)$} & \multicolumn{2}{|c|}{$E\left(4_{1}^{+}\right)$} & \multicolumn{2}{|c|}{$E\left(5_{1}^{+}\right)$} & \multicolumn{2}{|c|}{$E\left(6_{1}^{+}\right)$} \\
\hline & Teori & Deney & Teori & Deney & Teori & Deney & Teori & Deney & Teori & Deney \\
\hline${ }_{60}^{150} \mathrm{Nd}$ & 1043 & 1061 & 1158 & 1200 & 1311 & 1353 & 1503 & - & 1732 & - \\
\hline${ }_{64}^{152} G d$ & 1126 & 1109 & 1469 & 1434 & 1926 & 1550 & 2497 & 1861 & 3183 & 1998 \\
\hline${ }_{62}^{152} \mathrm{Sm}$ & 1079 & 1086 & 1195 & 1234 & 1351 & 1372 & 1546 & 1560 & 1780 & 1728 \\
\hline${ }_{66}^{154} D y$ & 1051 & 1027 & 1385 & 1334 & 1831 & 1443 & 2388 & 1740 & 3057 & 1885 \\
\hline${ }_{64}^{154} G d$ & 993 & 996 & 1112 & 1127 & 1270 & 1263 & 1468 & 1432 & 1705 & 1607 \\
\hline${ }_{62}^{154} \mathrm{Sm}$ & 1411 & 1440 & 1491 & 1539 & 1597 & 1665 & 1730 & 1805 & 1889 & 1946 \\
\hline${ }_{66}^{156} D y$ & 892 & 890 & 1025 & 1022 & 1203 & 1169 & 1426 & 1336 & 1693 & 1525 \\
\hline${ }_{68}^{156} \mathrm{Er}$ & 986 & 930 & 1332 & 1243 & 1793 & 1351 & 2370 & 1663 & 3062 & - \\
\hline${ }_{64}^{156} G d$ & 1129 & 1154 & 1214 & 1248 & 1328 & 1355 & 1470 & 1506 & 1641 & 1643 \\
\hline${ }_{66}^{158} D y$ & 929 & 946 & 1024 & 1044 & 1151 & 1164 & 1309 & 1315 & 1499 & 1486 \\
\hline${ }_{64}^{158} G d$ & 1156 & 1187 & 1232 & 1265 & 1334 & 1358 & 1461 & 1481 & 1613 & 1623 \\
\hline${ }_{68}^{160} \mathrm{Er}$ & 845 & 854 & 967 & 987 & 1332 & 1317 & 1858 & 1743 & 2546 & 2244 \\
\hline${ }_{64}^{160} G d$ & 953 & 988 & 1025 & 1057 & 1121 & 1148 & 1240 & 1261 & 1383 & 1392 \\
\hline${ }_{66}^{162} D y$ & 860 & 888 & 938 & 963 & 1041 & 1061 & 1169 & 1183 & 1324 & 1324 \\
\hline${ }_{68}^{162} \mathrm{Er}$ & 882 & 900 & 980 & 1002 & 1111 & 1128 & 1274 & 1286 & 1471 & 1460 \\
\hline${ }_{66}^{164} D y$ & 749 & 761 & 821 & 828 & 916 & 916 & 1035 & 1025 & 1178 & 1155 \\
\hline${ }_{68}^{164} \mathrm{Er}$ & 838 & 860 & 926 & 946 & 1043 & 1059 & 1189 & 1197 & 1365 & 1359 \\
\hline${ }_{70}^{166} Y b$ & 914 & 932 & 1012 & 1039 & 1144 & 1163 & 1308 & 1327 & 1505 & 1482 \\
\hline${ }_{72}^{168} \mathrm{Hf}$ & 864 & 875 & 984 & 1031 & 1143 & 1216 & 1343 & - & 1582 & - \\
\hline${ }_{70}^{168} \mathrm{Yb}$ & 959 & 983 & 1044 & 1067 & 1156 & 1171 & 1297 & 1302 & 1466 & 1445 \\
\hline
\end{tabular}

İncelenen çekirdeklerde enerjiyi minimum yapan $\beta$ değeri, deneysel olarak verilen birinci taban durumu $2_{1}^{+}$uyarılma enerjisi ile daha yüksek olan enerji bantlarından $\beta$ bandının $0_{2}^{+}$durum enerjisi veya $\gamma$ bandının $2_{3}^{+}$enerjisini en yakın şekilde doğru verebilmesi için ayarlanmıştır. Deneysel değerleri yakın bir 
biçimde tahmin edebilmek için tüm serbest parametreler hassas biçimde ayarlanmıştır. Tablo 1 den deformasyon parametresinin 0 ile $\sqrt{2}$ arasında olduğu görülmektedir. Ayrıca $\kappa^{\prime \prime}$ parametresi pozitif değer alırsa, diğer parametrelerin değerleri ne olursa olsun enerji minimumu daima 0 olmaktadır. Bu parametre belli bir negatif değerde minimum enerjiyi sıfırda verirken, belli bir değerin ötesine geçildiğinde faz geçişi gözlenir ve enerji minimumu $\sqrt{2}$ değerine doğru kayar. Bu parametrenin taban durum enerji yüzeyine etkisi farklı $\kappa$ "değerlerinde de değişmektedir.

\section{IV.SONUÇLAR}

Bu çalışmada, deneysel sonuçları belli olan A 160 civarındaki çift-çift çekirdeklerin Eşevreli Durum Yaklaşımı kullanılarak teorik taban durumu, beta ve gama faz geçiş düzeylerinin bulunması için serbest parametrelerin en iyi sonucu verecek şekilde değiştirilmesi üzerine bir çalışmadır. A 160 kütle numarasına sahip olan 20 çekirdek kullanılarak, her bir çekirdeğin bozon sayısı ayrı ayrı hesaplanarak en uygun Hamiltoniyen parametreleriyle en yakın sonuçlar bulunması amaçlanmışıı. Tablolar ve grafikler kullanılarak hesaplanan değerler karşılaştırılması olarak grafiklerle verilmiş olup hem de taban durum, Beta ve Gama düzeyleri için ilk beş enerji düzeyi tablolarla verilmiştir. Tablolar ve grafikler incelendiğinde son kapalı kabuğa yakın olan çekirdekler için; daha açık bir ifadeyle küreselliğe daha yakın olan çekirdekler için sonuçların daha tutarlı olduğu gözlenmektedir. Nükleon sayısı arttıkça çekirdeğin yüksek enerji düzeylerinde teorik hesaplarla deneysel sonuçların uyuşmaması dikkat çekmektedir. Düşük enerji düzeyleri için çekirdeğin enerji düzeyleri daha yakın sonuçlarla hesaplanmaktadır.

Özet olarak çalışmadaki çekirdeklerin Hamiltoniyenlerinde kullanılan parametreler arttıkça, deneysel sonuçlarla teorik hesapların uyuşma oranının daha yüksek olduğu görülmektedir. Kullanılan Hamiltoniyen yüksek enerji düzeyleri için farklı parametrelerle beraber kullanılması ile daha yakın sonuçlar vermesi üzerine tekrar çalışmalar yapılabilir. Çift-Çift çekirdeklerin enerji düzeylerindeki değişimin, nükleon sayısıyla olan iliş̧i üzerine yeni çalışmalar hazırlanabilir.

\section{KAYNAKLAR}

[1] Iachello, F. (2000). Physical Review Letter, 85, 3580-3583.

[2] Iachello, F. (2001). Physical Review Letter, 87, 052502.

[3] Ginocchio, J. N., Kirson, M. V. (1980). Relationship Between the Bohr Collective Hamiltonian and the Interacting Boson Model, Physical Review Letter, 44, 1744-1747.

[4] Arima, A., Iachello, F. (1976). Interacting Boson Model of Collective States I. The Vibrational Limit, Annals of Physics, 99, 253-317.

[5] Ginocchio, J. N., Kirson, M. V. (1980). Relationship Between the Bohr Collective Hamiltonian and the Interacting Boson Model, Nuclear Physics A, 350, 1744-1747.

[6] Arima, A., Iachello, F. (1976). Interacting Boson Model of Collective States II. The Vibrational Limit, Annals of Physics, 111, 201-138.

[7] Arima, A., Iachello, F. (1976). Interacting Boson Model of Collective States IV. The O(6) Limit, Annals of Physics, 123, 468-492.

[8] Khalaf, A. M., Okasha, M. D., Ahmed, G. S. M., Abdelsalam, A.(2020). Identical Bands in Doubly Even Nuclei in Framework of Variable Moment of Inertia (VMI) and Interacting Boson Models, Nuclear Physics A, 997, 121719.

[9] Iachello, F., Arima, A. (1987). The Interacting Boson Model, Cambridge University Press, Cambridge. 
[10] Inci, I., (2014). Test of Coherent State Approach in the Axially Deformed Region, Nucleer Physics A, 924, 74-83.

[11] Schaaser, H., Brink, D. M. (1984). Calculations Away From SU(3) Symmetry by Cranking the Interacting Boson Model, Physics Letters B, 143, 269-272.

[12] Bohr, A., Mottelson B. R. (1987). Nuclear Structure, vol. I-II, World Scientific, Singapore.

[13] Dieperink, A. E. L., Scholten, O. (1980). On Shapes and Shape Phase Transitions in the Interacting Boson Model, Nucleer Physics A, 346, 125-138.

[14] Ginocchio, J. N., Kirson, M. V. (1980). An Intrinsic State for the Interacting Boson Model and Its Relationship to the Bohr-Mottelson Model, Nucleer Physics A, 350, 31-60.

[15] Inci, I., Alonso, C. E., Arias, J. M., Fortunato, L., Vitturi, A. (2009). Coherent State Approach to the Interacting Boson Model: Test of Its Validity in the Transitonal Region, Physical Review C, 80, 034321.

[16] derMateosian, E., Tuli, J. K.(1995). Nuclear Data Sheets, National Nuclear Data Center Brookhaven National Laboratory, 75, 827-989.

[17] Reich, C. W. (2003). Science Direct, Nuclear Data Sheets 99, 753-1030.

[18] Helmer R. G. (2004). Science Direct, Nuclear Data Sheets 101, 325-519.

[19] Baglin, C. M. (2008). Science Direct, Nuclear Data Sheets 109, 1103-1382.

[20] Baglin, C. M. ( 2010). Science Direct, Nuclear Data Sheets 109, 1807-2079.

[21] Singh, B. (2002). Science Direct, Nuclear Data Sheets 95, 995-1036.

[22] Reich, C. W. (2009). Science Direct, Nuclear Data Sheets 110, 2257-2532.

[23] Reich, C. W. (2005). Science Direct, Nuclear Data Sheets 105, 557-774.

[24] Reich, C. W. (2007). Science Direct, Nuclear Data Sheets 108, 1807-2034.

[25] Singh, B. (2001). Science Direct, Nuclear Data Sheets 93, 243-446,

[26] Baglin, C. M. (2002). Science Direct, Nuclear Data Sheets 96, 611-874. 